\title{
Ueber die Abnahme der Sehschärfe im Alter.
}

\author{
Von \\ Prof. Hermann Cohn \\ in Breslau.
}

Mit 1 Textfigur.

In diesem Archiv (Bd. XXXIX, Abth. 2, S. 71) haben Dr. Boerma und Dr. Walther in Leipzig vor Kurzem Untersuchungen iber die Sehschärfe im Alter mitgetheilt. Sie hatten ein anderes Resultat gefunden, als Vroesom de Haan und Donders vor 30 Jahren.

Schon im Jahre 1874 habe ich auf der NaturforscherVersammlung zu Breslau eigne Untersuchungen ïber die genannte Frage veröffentlicht, welche das sogenannte de Haan'sche Gesetz umstiessen. Man findet meine Resultate kurz mitgetheilt unter dem Titel „Die Augen der Greise" im Tageblatt der Naturforscher-Versammlung zu Breslau vom 19. Sept. 1874, S. 105, ferner unter dem Titel "Untersuchungen der $\mathbf{S}$ in der Jugend und im Alter" als Bericht eines in der allgemeinen Versammlung der schlesischen Gesellschaft an 5. Februar 1875 gehaltenen Vortrages in der schlesischen Zeitung vom 21. u. 23. Febr. 1877, endlich im Auszuge in meinem Lehrbuche der Hygiene des Auges, Wien, 1892, S. 33. - Auch in Nagel's Jahrbuch für 1874, S. 210 und 222 und in Zehender's Monatsbl. f. Augenheilk. 1875, S. 79 findet man Referate. -

$\mathrm{Da}$ diese Untersuchungen gänzlich in Vergessenheit 
gerathen zu sein scheinen - weder Boerma und Walther noch die neueren Lehrbücher erwähnen sie -- so erlaube ich mir, hier auf dieselben zurïckzukommen, sie noch ausführlicher mitzutheilen, zumal sie auch die Refraction und die Augenleiden der Greise umfassen, und meine Befunde mit denen der genannten Leipziger Autoren zu vergleichen. -

Schon vor 20 Jahren labe ich auf die ungenügende Zahl und Auswahl der Personen hingewiesen, welche de Haan zur Aufstellung seines Gesetzes beniitzte. Fr prüfte im Ganzen 231 Personen von 7-82 Jahren, welche gerade: als Begleiter von Kranken in die Augenklinik von Donders kamen, und zwar mit den leichter erkennbaren Buchstaben von Snellen No. XX. Sah er eine Augenkrankheit, so wurde der Fall ausgeschlossen. „Alle Augen wurden sorgsam mit blossem Auge, viele auch mit dem Spiegel untersucht."

So wurde eine Curve construirt, uach der die Sehschärfe bis zum 30 . Tahre fast unveründert $\frac{22}{30}$ beträyt, in 50. Jahre auf $\frac{18}{20}$, im 60 . auf $\frac{14}{20}$, im 70 . auf $\frac{12}{20}$, im 80. aber auf die Hälfte herabsinkt. Die Ursachen dieser wenig tröstlichen Erscheinung suchte Donders zum Theil ,in geringerer Durchsichtigkeit der Linse und des Glaskörpers, zum Theil in noch unbekanuten Veränderungen in der Netzhaut, dem Sehnerven und Gehirn." Dies Gesetz war nur anf 281 Beobachtungen an Holländern basint. wurde aber Dogma für die ganze Welt; wir hatten Alle dieselbe traurige Perspective.

Der Satz blieb unangetastet bis 1871 , wo ich bereits die Mittelwerthe der $\mathbf{S}$ für die Jugend nach Untersuchungen der Augen der Schulkinder in Schreiberhau ganz anders fand als de Haan. Man vergleiche meinen Aufsatz in diesem Archiv, Bd. XVI, Abth. 2, S. 305. 
Viel wichtiger aber schien mir eine Revision des de Haan'schen Gesetzes für das Alter. Demn de Haan hatte nur 41 Personen über 60 Jahre untersucht, und unter diesen waren 13 Augenkranke, so dass also die Befunde an nur 28(!) Menschen die Grundlage des Gesetzes über die rapide Abnahme der $\mathbf{S}$ nach dem 60. Jahre bildeten. Ferner hatte er nicht alle Personen mit dem Augenspiegel untersucht; wir wissen also gar nicht, ob nicht bestimmte Netzhaut- oder Aderhaut-Erkrankungen die Sehschwächen erklärt haben würden. Daher schien mir schon vor 20 Jahren eine Revision der S alter Leute nöthig. Die Schwierigkeit lag aber damals wie heute in der Herbeischaffung des Materials. Denn selbst wenn man alle Personen, die über 60 Jahre sind, durch die Zeitungen zu einer Augen-Untersuchung einladen dürfte, würden ja doch viele ausbleiben ans Lethargie, aus Mangel an Zeit, an Verständniss etc.

Eine Reihe glücklicher Umstände vereinigte sich jedoch 1874, um mir die lang gewünschte Gelegenheit zu geben, die Frage wenigstens für einen Ort und zwar für Schreiberhau im Riesengebirge zu lösen. Dort hatte mir der Schulrector Herr W inkler und der Amtsvorsteher Herr Pohl den Weg geebnet. Man war von Haus zu Haus gegangen, hatte die alten Leute aufgesucht, über mein Vorhaben belehrt, die Alterstabelle entworfen und alle Personen über 60 Jahre für bestimmte Tage in bestimmte Gasthäuser des Ortes zu mir bestellt.

Mit Dr. Nobis und 2 Studenten begann ich am 1. Ang. 1874 die Arbeit und es gelang mir, als die Menge der sich vorstellenden alten Leute nach einigen Tagen spärlicher wurde, die Zahl 100 dadurch zu erreichen, dass ich das Erscheinen der alten Männer durch Bier oder Geld und das der alten Frauen durch Kaffee belohnte.

Schreiberhau war aber auch besonders für derartige Untersuchungen geeignet, weil der Kreis Hirschberg in Schlesien, in welchem Schreiberhau liegt, 9,1\% Personen über 60 Jahre enthält, während sonst in Preussen nur 6,8\% über 60 Jahre alt sind. In Preussen vertheilen sich die 
Letzteren so, dass $69 \%$ zwischen 60 und 70 Jahre, $27 \%$ zwischen 70 and 80 Jahre und $4 \%$ nur über 80 Jahre alt sind. Hingegen ist das Verhältniss in Schreiberhau : $58 \%$, $33 \%$ und $9 \%$. Von den 3681 Einwohnern des Gebirgsdorfes waren damals übrigens 328 Analphabeten $=9 \%$, die ihr Auge also gewiss nicht angestrengt hatten.

Ich prïfte von den 142 Uebersechzigjährigen 100 Personen, also $70 \%$, und zwar $70 \%$ derer von $60-69,77 \%$ derer von $70-79$ und $50 \%$ derer von $80-84$ Jahren. (104 Personen waren erschienen, doch konnten die Angaben von 4 Lenten wegen Taubheit und Schwachsinnigkeit nicht verwerthet werden).

Von 60 Männern wurden 43 , von 82 Weibern wurden 57 untersucht. 58 Personen waren zwischen 60 und 69,36 Personen zwischen 70 und 79 and 6 Personen zwischen 80 und 84 Jahr alt.

Die Prüfung wurde damals unter freiem Himmel mit der $\Pi$ Tafel von Snellen Nr. XX vorgenommen und durch Drehung der Zeile die Probe variirt; die Tages-Beleuchtung war stets eine sehr gute.

Nach der S-Probe wurde der Bau, der Farbensinn und die Accommodationsbreite, und in jedem der 200 Angen, Linse, Glaskörper, Netzhaut und Nerv mit dem Spiegel geprüft. Erst wenn drei Antworten über die Richtung der Oeffnung der Haken übereinstimmten, wurde der Befund der $\mathrm{S}$ notirt.

Die 100 Personen hatten folgende Refraction: $19 \mathrm{E}$, $49 \mathrm{H}, 9 \mathrm{M}, 5$ auf einem Auge $\mathrm{H}$, auf dem anderen unbestimmbar, 7 ein Auge $\mathrm{H}$, das andere $\mathrm{E}, 3$ ein Auge $\mathrm{M}$, das andere E, 4 ein Auge M, das andere H, 1 ein Auge E, das andere unbestimmbar, 3 überhaupt unbestimmbar.

In 12 Augen konnte die Refraction also nicht bestimmt werden und zwar wegen Ablösung der Netzhaut, Glaukom, Atrophia choroideae centralis, Maculae centrales, Cataract, Pannus trachomatosus und Pterygium. 49 Augen hatten E, $114 \mathrm{H}, 25 \mathrm{M}, 12$ unbestimmbar. Die $\mathrm{E}$ beider Augen betraf 27 Personen von $60-73$ Jahren: bei älteren Leuten wurde $\mathrm{E}$ nicht mehr gefunden. $\mathrm{E}$ eines und $\mathrm{H}$ des anderen Auges kam in 7 Fällen von $62-83$ Jahren vor.

Myopie beider Augen hatten 9 Personen von 61-83 Jahren und zwar:

$$
\text { Me } \frac{1}{60} \text { bis } M \frac{1}{11} \text {. }
$$


(Dié damaligen Proben wurden noch mit Zollgläsern gemacht, daher hier die Brüche). Bei 4 von diesen war die Linse klar bei

$$
\mathrm{M} \frac{1}{60}, \frac{1}{30} \text { und }-\frac{1}{16} \text {; }
$$

der Kranke mit $M \frac{1}{16}$ war von Jugend an Schreiber, die übrigen schwachen Myopen hatten unur häusliche Arbeiten gemacht. Bei den 5 anderen war eine Linsentrübung vorhanden.

M eines und $\mathrm{E}$ des anderen Auges kam 3 Mal vor, und zwar:

$$
\mathrm{M} \frac{1}{50}, \frac{1}{40} \text { und } \frac{1}{24}
$$

bei Leuten von 62-71. Jahren; einmal dabei Linsentrübung, einmal Macula, einmal chronischer Catarrh.

$\mathrm{M}$ eines und $\mathrm{H}$ des anderen Auges in 4 Fällen; in 2 Fällen zeigten beide Angen beginnenden Staar. Die Grade dieser $\mathrm{H}$ und $\mathrm{M}$ waren sehwach:

$\mathrm{H} \frac{1}{36}$ mit $\mathrm{M} \frac{1}{80}$ und $\mathrm{M} \frac{1}{36} ; \quad \mathrm{H} \frac{1}{80}$ mit $\mathrm{M} \frac{1}{50} ; \mathrm{H} \frac{1}{60}$ mit $\mathrm{M} \frac{1}{40}$. Im Ganzen waren unter $25 \mathrm{M}$-Augen 13 kataraktös. Der Durchschnittsgrad der M dieser 25 Augen war $\frac{1}{38}$; der Durchschnittsgrad der auf beiden Augen Myopischen war $\frac{1}{30}$.

Hyperopie beider Augen hatten 49 Personen; gleiche Grade von H 36 Personen. $H$ schwankte zwischen $H \frac{1}{11}$ bis $\frac{1}{80}$. Von $60-64$ Jahren war der Durchschnittsgrad $\mathrm{H} \frac{1}{43}$, von $65-69 \mathrm{H} \frac{1}{34}$, von $70-74 \mathrm{H} \frac{1}{46}$, von $75-80$ Jahren $\mathrm{H} \frac{1}{35}$. Von 5 zu 5 Jahren nimmt also die $H$ nicht zu. Der Durchschnittsgrad aller doppelseitig Hyperopischen war $\mathrm{H}=\frac{1}{39.8}$, der Durchschnittsgrad aller 114 hyperopischen Augen war $\mathrm{H}=\frac{\mathbf{1}}{37.8}$. 
Bei 13 Personen war der Grad der $\mathrm{H}$ auf beiden Augen verschieden. Der Durchschnittsgrad der schwächer hyperopischen dieser Augen war $\mathrm{H} \frac{1}{41}$, der der stärker hyperopischen H $\frac{1}{29}$; der durchschnittliche Unterschied in den Graden der $\mathrm{H}$ zwischen rechtem und linkem Auge betrug mithin $\frac{1}{29}-\frac{1}{41}$ $=\frac{1}{99}$; er war also sehr gering.

Die Accommodation war in einzelnen Fällen geradezu noch glänzend. So las Johanna Häusler, 70 Jahre alt, mit jedem Auge ohne Glas noch $\mathrm{I} \frac{\mathrm{I}}{\mathrm{II}}$ in $6 \mathrm{Zoll}$; sie hat $\mathrm{H} \frac{1}{36}$ und $\mathrm{S} \frac{32}{20}$. Ihre Mutter wurde 76 Jahre und nähete noch bis zu ihrem Tode ohne jede Brille. Der Vater wurde 84 Jahre und las noch kurz vor seinem Ende Monatsschriften ohne Brille. Von 12 Geschwistern leben noch 5, die sämmtlich ohne Brillen arbeiten.

Ferner hatte Barbara Schärf, 72 Jahre alt, für $I_{\text {II }}$ noch 6 Zoll Nahpunkt ohne Brille, dabei $\mathrm{E}$ und $\mathrm{S}^{28 / 20}$. Ihr Vater wurde 100 Jahre alt und brauchte keine Brille zum Lesen.

Endlich sei erwähnt Christiane Sender, 76 Jahre, die $H \frac{1}{12}$ und $\mathrm{S} 1$ zeigte und noch ohne Brille einfädelte.

Farbenblindheit wurde bei keinem Auge gefunden.

Die Sehschärfe der 200 Augen war:

$$
\begin{array}{lll}
88 \mathrm{~S}>20 / 20 & 34 \mathrm{~S}=2 \% \\
44 \% & 17 \% & 78 \mathrm{~S}<2 \% \\
39 \% & 39 \%
\end{array} \text { also }
$$

Von 100 binoculär geprüften Personen hatten 58 $\mathrm{S}>{ }^{20} / 20,12 \mathrm{~S}={ }^{20} / 20$ und $30 \mathrm{~S}<{ }^{20 / 20}$. Also $70 \%$ hatten binoculär keine herabgesetzte Sehschärfe, Das widerspricht völlig dem de $\mathrm{H}$ aan'schen Gesetze.

Augen mit $\mathrm{S}<1$. Es wurden 78 bei 46 Personen gefunden. Nur 10 Augen bei 5 Personen hatten eine unerklärbare Amblyopie. 68 Augen von 41 Personen mit $\mathrm{S}<1$ zeigten jedoch folgende Krankheiten: Katarrhus chronicus 1, Maculae corneae 3, Keratitis interstitialis 2, Pannus tracho- 
matosus 2, Pterygium magnum 3, Synechiae posteriores 2, Katarakt 27, Neuroretinitis 1, Retinitis albuminurica 2, ChorioRetinitis 4, Hyperaemia optiei 2, Atrophia optici 14, Sublatio retinae traumatica 1, Glaucom 3, Nystagmus 1.

Bei 14 Personen war nur ein Auge erkrankt, bei 27 Personen beide. Atrophia optici mit Katarakt wurde im Ganzen 11 mal gesehen.

Ausserdem zeigten noch 34 Augen-Krankheiten, ohne dass dadurch $\mathrm{S}$ gelitten hätte, u. zw. Cornealflecke 3, Pterygium 1, beginnende Katarakt 20, Katarrh 3, Glaucoma chronicum 1, Dacryocystoblennorrhoe 4, Neuroretinitis 1, Ptosis 1.

6 mal sah ich sehr tiofo physiologische Excavation ohne Verschlechterung der Sehschärfe.

$\mathrm{S}<1$ fand sich bei 7 Krankheiten der Bindehaut, 8 der Hornhaut, 2 der Iris, 33 der Linse, 11 der Aderhaut, 23 der Netzhaut, 2 der Muskeln, 2 der Thränenorgane, 3 der Lider und 3 Verletzungen; also $94 \mathrm{Krankheiten}$ bei 68 Augen von 41 Personen.

Ringförmige Aderhaut-Atrophie sah ich 3 mal bei guter $\mathrm{S}$ und $3 \mathrm{mal}$ bei herabgesetzter $\mathrm{S}$. Verkalkungen der Meibom'schen Drüsen kamen häufig vor.

Berechnet man nach Procenten, so vertheilen sich die Krankheiten der 41 Personen so: Vom 60.-64. Jahre 22\%, vom 65.-69. $37 \%$, vom $70 .-75$. 50\%, vom 75.-79. Jahre $83 \%$. und vom 80.-84 Jahre $66 \%$. Mit den zunehmenden Jahren ist also die Wahrscheinlichkeit der Erkrankung eine grössere.

Katarakt fand ich in 53 Augen, davon hatten 33 Verschlechterung der $\mathrm{S}, 20$ jedoch $\mathrm{S}=1$ oder sogar $>1$. Der vierte Theil aller Augen, die uber $60 \mathrm{Jahre}$ alt waren, zeigte Katarakt. Die 53 Angen gehörten 32 Personen; 21 hatten doppelseitige, 11 nur einseitige Trübungen. 12 Augen waren E, $28 \mathrm{H}$ und $13 \mathrm{M}$. Letztere gehörten 8 Personen; bei 5 derselben war $\mathrm{M}$ und Katarakt beiderseitig; bei 3 Personen einseitig. Die Katarakte betrafen 13 Männer $(30 \%)$ und 19 Frauen $(33 \%$ der Untersuchten).

Katarakt batten $20 \%$ von $60-69$ Jahren, $42 \%$ von 70-79 Jahren, $100 \%$ von 80-84 Jahren. Der Staar wird also von Jahrzehnt zu Jahrzehnt häufiger.

Unter 20 kataraktösen Augen, die keine schlechte $\mathrm{S}$ zeigten, waren sogar 11 mit $S>1$ und zwar mit $S^{21 / 20}$ bis 
$\mathrm{S}^{30} / 20$. Eine Kranke mit $\mathrm{S}^{28} / 20$ war 73 Jahre, ein Kranker mit $\mathrm{S}^{30} / 20$ auf beiden Augen war 83 Jahre! Die Trübung ragte eben erst als zarte Streifchen in einigen Sectoren über den Pupillarrand hinweg.

\section{Augen mit $S>1$.}

88 Augen zeigten eine solche, und zwar hatten 70 Augen $\mathrm{S}>1$ bis $\mathrm{S}^{3} / 2,17$ Augen $\mathrm{S}>3 / 2$ bis 2,1 Auge $\mathrm{S}^{42 / 20}$ d. $\mathrm{h}$. $\mathrm{S}>2$.

$\mathrm{S}>1$ zeigten $57 \%$ der Augen von 60-69 Jahren, $28 \%$ von $70-79$ Jahren and $33 \%$ von $80-84$ Jahren. Im 7. Jahrzehnt sind also die übergrossen Sehschärfen noch häufiger als in den späteren Jahrzehnten.

Die folgenden Tabellen geben über die Sehschärfen im Ganzen Aufschluss.

\begin{tabular}{|c|c|c|c|c|c|c|c|c|}
\hline $\begin{array}{l}\text { Untersuchte } \\
\text { Angen }\end{array}$ & Alter & $s=1$ & $\mathrm{~s}=\frac{21-25}{20}$ & $\frac{26-30}{20}$ & $\frac{31-35}{20}$ & $\frac{36-40}{20}$ & $\begin{array}{c}\mathrm{S}<1 \\
\text { ohne } \\
\text { patho- } \\
\text { logisch. } \\
\text { Befnad, }\end{array}$ & $\begin{array}{c}s<1 \\
\text { mit } \\
\text { patho- } \\
\text { logisch. } \\
\text { Befund. }\end{array}$ \\
\hline $\begin{array}{l}62 \\
54 \\
60 \\
12 \\
12 \\
\end{array}$ & $\begin{array}{l}60-64 \\
65-69 \\
70-74 \\
75-79 \\
80-84\end{array}$ & $\begin{array}{c}12 \\
4 \\
14 \\
3 \\
1\end{array}$ & $\begin{array}{c}18 \\
8 \\
2 \\
2\end{array}$ & $\begin{array}{l}11 \\
15 \\
12 \\
\frac{2}{2}\end{array}$ & $\begin{array}{l}7 \\
4 \\
1 \\
- \\
-\end{array}$ & $\begin{array}{l}1 \\
3 \\
2 \\
- \\
-\end{array}$ & $\begin{array}{l}4 \\
2 \\
4 \\
- \\
\end{array}$ & $\begin{array}{c}9 \\
18 \\
25 \\
9 \\
7\end{array}$ \\
\hline 200 Augen & $60-84$ & 34 & 30 & 40 & 12 & 6 & 10 & 68 \\
\hline $\begin{array}{l}\text { Unter } \\
100 \text { Augen } \\
\text { ron }\end{array}$ & $\begin{array}{c}s= \\
\frac{40-36}{20}\end{array}$ & $\begin{array}{c}5= \\
\frac{35-31}{20}\end{array}$ & $\frac{30-36}{20}$ & $\left|\frac{25-21}{20}\right|$ & $S=80,20$ & $\begin{array}{c}S<1 \\
\text { Ambl. }\end{array}$ & $\begin{array}{c}\mathrm{s}<1 \\
\text { Augen } \\
\text { krank }\end{array}$ & || Summa \\
\hline $\begin{array}{l}60-64 \text { Jahr. } \\
65-69 \quad " \\
70-74 \quad " \\
75-79 \quad " \\
80-84 \quad "\end{array}$ & $\begin{array}{l}1 \\
5 \\
2 \\
- \\
-\end{array}$ & $\begin{array}{c}11 \\
7 \\
2 \\
- \\
-\end{array}$ & $\begin{array}{l}18 \\
28 \\
20 \\
16\end{array}$ & $\begin{array}{c}29 \\
15 \\
4 \\
16\end{array}$ & $\begin{array}{c}19 \\
7 \\
24 \\
25 \\
8\end{array}$ & $\begin{array}{l}7 \\
4 \\
6 \\
- \\
\end{array}$ & $\begin{array}{l}15 \\
34 \\
42 \\
75 \\
60\end{array}$ & $\begin{array}{l}100 \\
100 \\
100 \\
100 \\
100\end{array}$ \\
\hline
\end{tabular}

Nach diesen Befunden sprach ich vor 20 Jahren schon die Ansicht aus, dass unsre Perspective für die $\mathbf{S}$ im Alter viel tröstlicher sei, als man bisher glaubte. - - -

Bereits 1872 hatte Mauthner (Vorlesungen über die 
optischen Fehler des Auges S. 139 und 147) auf die unzureichende Beobachtungsreihe Vroesom de Haans hingewiesen. „Namentlich möchten wir, ruft Mauthner, die $S$, die er 70 und 80 jährigen vindicirt, als pathologisch gering ansehen. Einfach in Folge des Alters sinkt $\mathrm{S}$ mit 80 Jahren kaum auf die Hälfte; gesunde Augen solcher Greise zeigen, soweit mein verhältnissmässig kleines Beohachtungs-Material lehrt $S \frac{15}{20}$; doch entscheiden können endgiltig nur die Massen."

Auch ich schloss meinen Vortrag auf der NaturforscherVersammlung 1874 mit den Worten: „Freilich gilt mein Befund nur für Schreiberhan, und ich bin weit entfernt, ihn zu generalisiren. Wollen wir wirklich ein Gesetz finden, so müssen solche Untersuchungen auf dem Lande und in den Städten, bei civilisirten und bei nicht civilisirten Völkerstämmen, bei verschiedenen Nationen, bei verschiedenen Berufsklassen, in den Thälern und auf den Bergen bei grossen Massen von Menschen angestellt werden. Möchten bald andere Fachmänner diesen meinen ersten schwachen Versuch, Klarheit ïber den Einfluss des Alters auf die Sehschärfe zu erhalten, wiederholen und nicht vor den Schwierigkeiten zuriickschrecken!"

Seit 19 Jahren hat sich aber Niemand an die Arbeit gemacht. Um so dankenswerther sind die neuen Untersuchungen vou Boerma und Walther. Sie konnten mit Westiens Lupe und mit dem Lupenspiegel noch mehr Feinheiten betr. der Trübung der Medien feststellen, als ich im Jahre 1874, wo diese Hilfsmittel unbekannt waren. Auch waren sie durch Anwendung von Homatropin, das ja auch damals noch nicht existirte, in der Lage, die Pupille vorübergehend zu erweitern. Dies hätte ich durch Atropin bei den Greisen in Schreiberhau nicht riskiren dürfen, ohne dass die Atropinisirten die Nachfolgenden vor der Untersuchung gewarnt hätten. 
Würde ich diese Hilfsmittel gehabt haben, so würde ich wahrscheinlich unter meinen 200 Augen auch bei den 10 mit $S<1$ pathologische Störungen gesehen haben, die mir entgingen, so dass die Zahl der Amblyopien ohne Befund ganz oder theilweise weggefallen wäre. Freilich war das Material von Boerma und Walther ein ganz anderes, als das meinige. Jene Forscher prïften meist Gefangene und Reconvalescenten und betonen selbst die zeitige Senescenz der Mehrzahl in Folge ihrer bewegten Vergangenheit, Noth und des Alkoholmissbrauchs. Die Greise in Schreiberhau waren dagegen wohl arm, aber gesund und keine Trinker. - Die 400 Leute in Leipzig waren 40 bis 80 Jahre alt. $\mathrm{H}>3 \mathrm{D}, \mathrm{M}>5 \mathrm{D}$ und $\mathrm{As}>1 \mathrm{D}$ wurde ilusgeschlossen; nur bei Leuten über 70 Jahren wurde auch $\mathrm{H}=4 \mathrm{D}$ zugelassen. Ich dagegen habe uiberhaupt keine $\mathrm{H}>3$ und keine $\mathrm{M}>2.5$ gefunden. - Auch die

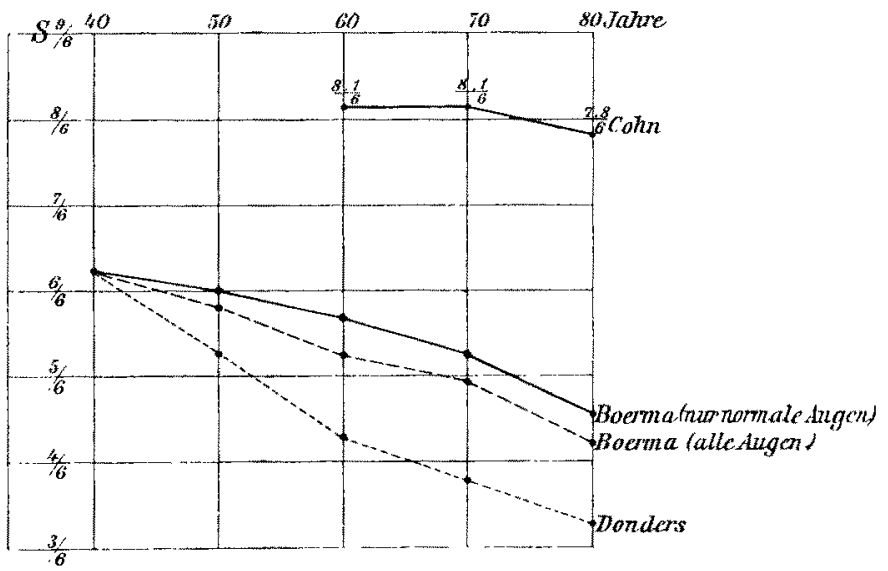

Fig. 1. Curvo.

Beleuchtungsverhältnisse waren in Leipzig ganz andere, als in Schreiberhau; in den verschiedensten Lokalen wurde dort bei verschiedener Witterung auf Snellen untersucht; ich liess dagegen an sehr hellen Tagen unter freiem 
336 H. Cohn. Ueber die Abnahme der Sehschärte im Alter.

Himmel Nr. XX Snellen lesen und zwar Haken. Daher erklären sich gewiss auch zum Theil die viel grösseren Schreiberhauer Sehschärfen.

Trotz alledem ist es immerhin interessant, de Haan's, Boerma's und meine Curve der Sehschärfen-Abnahme im Alter zu vergleichen.

Die Durchschnittsgrösse der $\mathbb{S}$ im 60. Jahre betrug in Schreiberhau $\frac{27}{20}=\frac{8.1}{6}$, im 70. Jahre ebensoviel und im 80. Jahre $\frac{26}{20}=\frac{7.8}{6}$, Sie betrug in Leipzig bei Ausschluss der pathologisch veränderten Augen im 40. Jahre $\frac{6.1}{6}$, im 50. Jahre $\frac{5.9}{6}$, im 60. Jahre $\frac{5.6}{6}$, im 70. Jahre $\frac{5.2}{6}$ und im 80. Jahre $\frac{4.5}{6}$. Nach Donders aber wäre sie in den genannten Jahrzehnten $\frac{6.1}{6}, \frac{5.4}{6}, \frac{4.3}{6}, \frac{3.9}{6}$, und $\frac{3.3}{6}$. Wie vieler Untersuchungen wird es noch bedürfen, um das wirkliche Gesetz der Abnahme der Sehschärfe im Alter zu finden! 